Ocean Sci., 6, 51-60, 2010

www.ocean-sci.net/6/51/2010/

(C) Author(s) 2010. This work is distributed under

the Creative Commons Attribution 3.0 License.

\title{
What are "ecogenomic sensors?" A review and thoughts for the future
}

\author{
C. A. Scholin \\ Monterey Bay Aquarium Research Institue, 7700 Sandholdt Road, Moss Landing, CA 95039, USA \\ Received: 10 June 2008 - Published in Ocean Sci. Discuss.: 30 January 2009 \\ Revised: 13 November 2009 - Accepted: 22 December 2009 - Published: 26 January 2010
}

\begin{abstract}
The OceanSensors08 workshop held 31 March4 April 2008 in Warnemünde, Germany, brought together an international group of marine scientists, sensor developers and technologists with a common interest in shaping the future of ocean sensing. In preparation for that meeting a series of review papers was commissioned, one of which was meant to cover Sensors for Ocean-omics. The "ocean-omics" topic was cast very broadly. The notion was to review use of genetic techniques for assessing presence and diversity of organisms, their genomic capacity and gene expression, and to provide a prospectus of how such methods could be applied in an autonomous capacity. I chose "ecogenomic sensor" as a descriptor to convey the essence of such a system - a device that integrates genetic level sensing with larger scale environmental characterization. This phrase is derived from workshops refining the US's Ocean Observatories Initiative (OOI) and visions for instrument systems that could be deployed on such a network. But what exactly are ecogenomic sensors? A clear definition is lacking and conceptualizations far outweigh actual hardware that can be deployed in the ocean. This prospectus builds from that point. I advance a definition of "ecogenomic sensor" and outline the opportunities and challenges associated with developing such instruments. Suggestions as to how this technology may be further refined and applied are offered against the backdrop of the Autonomous Microbial Genosensor (AMG) and Environmental Sample Processor (ESP). Applications that center on detection of DNA and RNA are emphasized. The word "review" appears in the title at the request of the editors.
\end{abstract}

\section{Correspondence to: C. A. Scholin} (scholin@mbari.org)

\section{Introduction}

An internet search using the phrase "ecogenomic sensor" will return numerous references that speak broadly to the idea of detecting molecular markers indicative of specific organisms, genes or other biomarkers within an environmental context. A strict and unified definition of "ecogenomic sensor" is lacking, however, and the phrase is used commonly for laboratory-based tools and techniques as well as semi or fully autonomous systems that can be deployed outside of the laboratory. Here I consider an ecogenomic sensor from the perspective of a field-portable device applied towards oceanographic research or water quality monitoring. It is a class of instrument that employs wet chemistry molecular analytical techniques to assess the presence and abundance of specified set of organisms, their genes and/or metabolites in near real-time. A broad definition of an ecogenomic sensor would naturally include devices capable of carrying out basic exploratory work as well, for example application of high density hierarchical probe arrays to deduce community structure and infer presence of species/genes not previously described, or even to conduct sequencing of DNA and RNA in situ.

For the purposes of the OceanSensors08 workshop, the premise was to consider development of a device that would sense molecular signatures that are already known, particularly defined sequences of DNA or RNA. This restricted view of an ecogenomic sensor was emphasized since prototypes of such instruments already exist and so provide a valuable benchmark for assessing the challenges of engineering and operating such "basic", deployable systems. Ecogenomic sensors as considered here would thus be designed to observe sets of defined biomolecular signatures in time-series fashion, and could also include sample archival functionality for supporting discovery work post-deployment. Furthermore,

Published by Copernicus Publications on behalf of the European Geosciences Union. 
information gleaned from such devices would necessarily be viewed within a larger observatory framework. Knowledge of the environmental conditions that prevailed at the time of sampling is essential if the molecular signatures detected and quantified are to be interpreted in an appropriate manner. Descriptions of the suite of sensors providing that framework are reviewed by others (e.g., OceanSensors08, 2008). At a minimum, an essential observatory backbone would include basic chemical, physical and bulk biological assessments such as temperature, salinity, pressure, chlorophyll fluorescence, etc. In that sense my definition of ecogenomic sensor parallels and narrows concepts outlined by Doney et al. (2004), Devereux et al. (2005), Bowler et al. (2009) and the "sensor robot" vision as illustrated in Fig. 1.

\section{Molecular markers as sensing elements}

The study of aquatic organisms at the molecular level has been a focus of research for decades. Subjects span environments from all corners of the globe, the shallowest of waters to the deep-sea, cold seeps to hot vents. Target organisms span an equally impressive range - viruses and phage to the largest marine mammals. The ready availability and reliability of commercially available instruments, methods and supplies has made it possible to dig into the molecular underpinnings of just about everything that is "environmental". The biotechnology revolution has played a key role in that regard. Most recently this has come to include genomics and associated technologies (e.g., Venter et al., 2004; DeLong et al., 2006; Giavannoni et al., 2008; DeLong, 2009 and others cited therein). These achievements, coupled with advances in ocean observatory technology and plans to extend those networks, have fueled the idea of applying "molecular sensors" in remote settings (Sandifer, 2007; HARRNESS Anonymous, 2007; Bowler et al., 2009). Hence, the notion of ecogenomic sensors as an emergent technology is fast becoming engrained within the ocean science psyche even though there is no formal agreement on what that phrase means. The evolution and application of ecogenomic sensors seems as natural as instruments that have brought real-time chemical, physical and bio-optical assessments of the ocean to our desks at a mere click of button. The picture is rosy - the technological leap required to achieve comprehensive ecogenomic sensing appears straightforward and timely.

Sweet as that dream may be, precious few examples of ecogenomic sensors exist and many longstanding challenges remain largely unmet. At present, for the majority of ocean research and monitoring programs, it is often not possible, practical nor cost effective to use conventional molecular analytical methods and equipment while in the field. Instead the vast majority of work generally occurs in specially equipped laboratories after the return of a discrete set of samples, limiting opportunities to collect molecular analytical data in near real-time time. Combined with sampling opportunities that are limited by financial, logistical or ship scheduling con- straints, 4-D (space-time) views of the environment from a molecular analytical perspective are often done retrospectively and intermittently. The reality of ecogenomic sensors is thus far removed from that implied in Fig. 1. Yet the image and underlying concepts do accurately reflect systems under development to some degree. The image also captures the requirement to embed such sensors within a broad ocean observatory framework.

\section{In the footsteps of biomedicine: the quest for ecogenomic sensors}

Zehr et al. (2009) provide a comprehensive summary of the application of molecular analytical techniques for environmental research. Other useful, recent reviews that serve as background information for this article include Devereux (2006), Metfies et al. (2006), Paul et al. (2007), Goodwin and Litaker (2008), Scholin et al. (2008) and DeLong (2009). These contributions and many others delve into the details of experimental concepts, tools and techniques, and the advantages and limitations of specific methodologies. Such details are not repeated here. Instead this contribution emphasizes the functional requirements for detecting specific microorganisms and the genes they harbor and express, and some of the challenges faced when projecting the development and use of novel instruments that will utilize these techniques onboard autonomous platforms. The views expressed are intentionally broad and draw from both academic and commercial sectors, and were meant to spur discussion at the OceanSensors08 workshop.

The development of molecular diagnostic equipment that is portable, battery operated and requires little training to use is a major focus of research in support of the biomedical industry. "Point-of-care-diagnostics" promises to make biomedical tests far smaller and easier to use than they are at present, and this will impact options for developing biosensors with ocean science applications (e.g., Metfies et al., 2006; LeGier et al., 2007). One of the real miracles of modern molecular biology is the ability to detect specific molecules at fleetingly low concentrations on a $\mu \mathrm{l}$, even $\mathrm{nl}$ scale. This has been a boon for biomedical applications given the tremendous advantage of requiring little sample material (drop of blood, skin swab). This makes integration of sample collection, processing and analytical functions in very small form factor possible.

Ecogenomic sensors rest fundamentally on the same ideals, but within the environmental domain sampling presents a much greater challenge. Many organisms or genes of interest may be relatively rare, requiring sample volumes of 100 's of ml to ensure that at least a few target cells are captured. More practical by today's standards are sample volumes of many L's to acquire a representative group of organisms from a given location at any one instant, and to accommodate vagaries of sample processing (Goodwin and Litaker, 


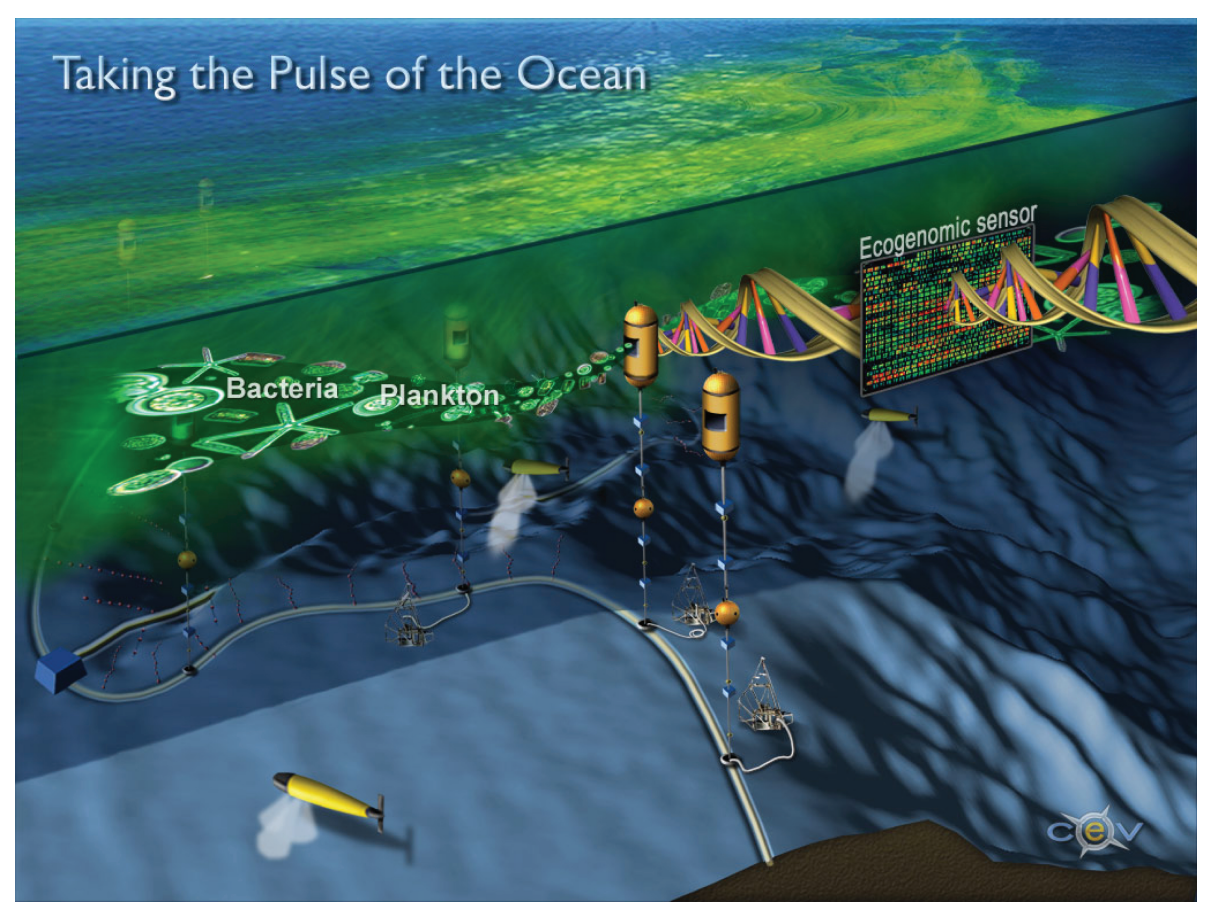

Fig. 1. Artist's rendition of ecogenomic sensors embedded within an array of airborne and in-water sensors. This image was cast in the broader vision of the US Ocean Observatories Initiative (OOI). Image courtesy of Dr. Virginia Armbrust, the Regional Scale Nodes Program, and the Center for Environmental Visualization, all at the University of Washington (after Armbrust et al., Report on the Ocean Genomics Workshop, http://www.neptune.washington.edu/research/index.jsp?keywords=ECOGNM $\backslash \&$ title=Ecogenomics .

2008). The relative abundance of the target organism, or target molecule, compared to the matrix of material within which those targets are embedded can also vary greatly in environmental samples. The size range of particles encountered in natural samples is another important consideration. Organisms of interest in the natural environment may be freeliving or found in aggregates, or both, further impacting the design of systems for automating sample collection and sample processing prior to final analysis (e.g., Lyons et al., 2007; Palmer et al., 2008). Many investigations targeting microbes apply a pre-filtration step to remove particles greater than $\sim 1 \mu \mathrm{m}$ prior to further analysis. Applying the same technique in an autonomous mode is not necessarily trivial. Consequently, working to overcome problems associated with sample acquisition and handling - the "front end" - should be viewed as an important step towards achieving an ecogenomic sensing capability. Such seemingly mundane needs are often overlooked in favor of the more exciting challenge of developing and applying the "back end" molecular detection schemes themselves. This situation is reflected in the disparity between technologies available for collecting samples from the ocean versus those available for sample analysis in a laboratory.

As summarized by authors cited earlier, molecular analyses focus on both intact cells and subcelluar fractions. Typically, particles in a sample are concentrated and then a series of reagents applied in a time and temperature depen- dent fashion. Extraction chemistries may be invoked to purify particular fractions prior to analysis. Target molecules may be detected solely on the basis of their inherent physical properties, or revealed using a variety of intermolecular reactions, such as antibody/antigen recognition, nucleic acid hybridization and enzyme mediated processes. Signal transduction and quantification most commonly involve optical techniques, though electrochemistry and mass spectroscopy are also employed.

In the laboratory, different steps associated with sample processing (collection, concentration, extraction, analysis) are generally accomplished using different pieces of equipment. Some companies offer laboratory and field portable instruments that meet some or all of these needs (e.g., Luminex, Cepheid, Gyros, Idaho Technology, Enigma Diagnostics, microfluidic ChipShop), but for the most part those systems are designed with very specific biomedical research and "biothreat" diagnostic applications in mind. As far as I am aware only the Autonomous Microbial Genosensor (AMG) and the Environmental Sample Processor (ESP), discussed in greater detail later, are designed to conduct cell-free molecular analyses in the ocean remotely (Paul et al., 2007; Scholin et al., 2009). Both adhere to fundamental sample processing schemes that parallel procedures used in laboratories. A discussion of these basic methods follows. In addition, other commercially available technologies that are not designed to be part of autonomous ocean sensors, but that could be 
applied to ocean research and monitoring, are noted. The focus here is analysis of nucleic acids free in solution following cell lysis, though generically most of the same requirements apply to analysis of other cell fractions too (e.g., proteins, cell metabolites).

\subsection{Sample collection and handling}

Tangential and direct flow filtration techniques are used commonly to acquire marine microbes from cultured and natural samples. Tangential flow filtration (TFF) applies the sample parallel to the filter media. Material passing through the membrane is removed (permeate) while the remainder (retentate) is recirculated back to the sample reservoir. This approach offers the advantages of concentrating small cells and viruses from very large sample volumes and the filter is reusable. However, TFF membranes are prone to fouling and require maintenance. Direct flow filtration (DFF) applies the sample perpendicular to the membrane to separate permeate and retentate. Membrane adsorption of particles based on electrostatic interactions is yet another approach for concentrating target microbes (see Palmer et al.(2008) for a comprehensive review of sample collection methods, and the Pall Corporation for a review of TFF and DFF theory and application). Relative to TFF, filters used for DFF handle less sample volume and are generally not reused. Though problems associated with fouling are greatly diminished, there is a requirement for exchanging filter media for each discrete sample. Some filter media can be used for both sample concentration as well for solid phase extraction chemistries (e.g., glass beads or glass fiber filters), making it possible to colocate the steps of acquiring and fractionating material ahead of analysis. Although the latter approach is attractive, it is not employed commonly. A variation of the DFF is the "renewable filter". This system has been applied to automate a variety of analyses for pathogen detection (Straub et al., 2005; Bruckner-Lea, 2000). It is not clear if the renewable filter concept can be extended to handle large sample volumes typically needed for analysis of marine samples; this warrants further investigation.

Regardless of whether TFF or DFF is applied, collection methods used for analysis of marine microbes generally yield a series of discrete samples, each representing a volume of water collected at a particular time, location and depth. In the laboratory sets of samples are often processed in batch mode. There may be a significant time lag between sample collection and analysis, but once analysis is initiated a very large amount of data can be generated very quickly. This paradigm is not well suited for autonomous systems if near real-time data are desired. Ideally, autonomous systems should be capable of processing samples serially immediately after collection, and in turn pass appropriate fractions to one or more molecular analyses downstream whilst initiating a new sample collection event. Molecular analytical determinations would thus emerge one sample at a time, and at a relatively slow rate (per hour) compared to rapid (per minute) chemical, physical and bulk biological assessments typical of present day observing systems.

\subsection{The case for microfluidics and sample homogenization}

Achieving autonomous time-series measurements using molecular techniques rests with accommodating relatively large sample volumes at the "front end" and supporting chemistries that require or greatly benefit from small scale reactions at the "back end". Most of the analytical reagents employed are purchased in bulk and can be expensive, but when used in $\mu \mathrm{l}$ quantities (or less) are cost-effective on a per sample basis. For example, one might spend hundreds of US \$'s in supplies to run PCR reactions, but when amortized over many samples expenditures are very reasonable (e.g., US \$5 per sample).

At the time of this writing, homogenizing cells and subjecting small, concentrated aliquots of that material to various analytical procedures appears to offer the most options for porting different molecular analyses from the laboratory to in situ autonomous systems. This is not to imply that molecular analyses based on intact cells are without merit (e.g., Varshney, 2007), but rather reflects a theoretical and generic "bang for the buck" argument given the current problems of bridging the gap between collecting samples from the marine environment, and carrying out a wide range of molecular analyses. One notable exception is the work by Ottesen et al. (2006) where highly parallel single cell analyses were demonstrated. Sample homogenization can be achieved by physical (e.g., sonication; Chandler et al., 2001) or chemical/physical techniques (e.g., detergent, heat; Greenfield et al., 2008), either of which can be built in to autonomous systems. The details of how and when these different approaches are applied in the laboratory vary widely, and to some extent are driven by whether molecular fractionation/purification is required ahead of analysis or if direct analysis of the crude homogenate will suffice.

Methods that rely on nucleic acid amplification offer the most sensitive assays for detecting low levels of target sequences (e.g., see Zehr et al., 2009; Frias-Lopez. 2008). These methods generally demand purified templates, though in some cases it may be possible to use crude homogenates or whole cells directly. Reaction mixtures are built up by adding a suite of reagents supplied in liquid or dehydrated form, the resulting cocktail is subjected to an appropriate thermal profile, and the reaction is often complete within $1 \mathrm{~h}$. With the exception of the initial sample collection/concentration step, these methods employ reagents and equipment that are well supported by commercial vendors. For example, Cepheid offers a self-contained laboratory system - the GeneXpert - for processing samples and applying quantitative PCR (qPCR). Enigma Diagnostics' laboratory based and field portable systems are very similar. In many ways the core functionality of 
the AMG parallels these systems. However, the GeneXpert and Enigma devices are designed to meet clinical microbiology and biothreat surveillance markets. These commercially available systems do have potential for enhancing environmental research and monitoring, but developing the assays and producing the specialized reagent cartridges needed for their use would be very costly compared to their market potential.

Less developed are methods that allow for direct analysis of target molecules without a requirement for amplification. This can be achieved by retaining target molecules on a solid support, or reacting probes with target molecules in solution (e.g. Bavykin et al., 2001; Ahn et al., 2006; Greenfield et al., 2008; Haywood et al., 2007; Jones et al., 2007; Dhadwal et al., 2007; Duy and Connell, 2007). Direct detection offers the advantage of reducing complexity of the detection chemistry, but such methods do not offer the same sensitivity for a wide range of target molecules as can be achieved when amplification techniques are applied. Instead, direct detection may be best applied for detecting highly abundant molecules, such as rRNA (Preston et al., 2009). When there is a need to detect low copy number targets, then alternative, albeit more demanding, methodologies such as those that invoke nucleic acid amplification may be required.

\subsection{The search for multiple molecular markers contained in a single sample}

For many environmental applications it is desirable to assess the presence of more than one target in a single sample. That is accomplished now using quantitative nucleic acid amplification techniques or through the application of probe arrays. In the laboratory, quantitative, amplification-based assays targeting multiple targets are often carried out in limited multiplexed reactions (detection of multiple targets in a single reaction). Detection of multiple markers can also be done in parallel (e.g., multi-well plates), with appropriate controls and dilution series evaluated in a single run. Parallel processing on scales routinely applied in the laboratory (e.g., 96 and 384-well plates) could be difficult to achieve aboard autonomous ocean sensors in the near future. Instead, in the near term, it seems more likely that a limited number of reactions will be run at the same time (e.g., 2-10) and reactions carried out serially (e.g., after Belgrader et al., 2003; Fukuba et al., 2004). By repeated cycles, it would be possible to build up a set of measurements for a given sample. But even with multiplexed reactions it could take hours to complete 10's of different reactions. Using probe arrays in concert with direct capture and amplification strategies may help alleviate some of those restrictions (e.g., Bevykin, 2001; Greenfield et al., 2008; Jones et al., 2007; Rich et al., 2007). Another way to overcome the limits imposed by carrying out PCR reactions by more conventional techniques is to utilize a microfluidic PCR array device such as that made by Fluidigm. One of their systems is capable of performing over 9000 real time
PCR reactions at the same time in a single chip (96 assays against 96 samples), and reportedly requires less pipetting to set up than is required for a 384-well plate format. These chips and supporting hardware have been adapted for detection of multiple genes from single bacterial cells (Ottesen et al., 2006). It is not clear if such systems can be adopted for use aboard an autonomous platform operating in the sea.

Probe arrays offer a means of detecting a large number of target sequences in a single sample simultaneously. However, current methods typically require extensive, sample preparation procedures to obtain labeled and amplified material that is suitable for analysis. Currently, the most widely used format is immobilized oligonucleotides on glass slides (e.g., Rich et al., 2007; see also Agilent). Microbead arrays on fiber optic bundles (Ahn et al., 2006; Illumina), and flowthrough systems that employ small, functionalized particles (Ellison and Burton, 2005, Luminex) are also highly developed formats. STMicroelectonics offers the In-Check platform, a microfluidic chip that combines PCR amplification and probe array detection functions. As noted earlier, Fluidigm's microfluidic dynamic arrays offer similar options for combining sample handling and analysis. Integrated devices like those could find application for deploying "conventional probe array chemistries" in an ocean setting so long as the upstream sample collection and handling are met by some other means. This poses significant, though not necessarily insurmountable, challenges for an autonomous system deployed at sea. Direct detection of target molecules in an array format without amplification is also feasible as noted earlier and described below.

\subsection{Reagents storage and stability}

Regardless of the approach chosen, all of the techniques outlined above use expendable reagents. Field portable systems that automate these methods, whether "hand held" or "embedded", require a ready supply of consumables and flush fluids for keeping the instrument clean. If single-use "chips" (microarrays or integrated fluidic devices) are employed, they too must be exchanged. Thus, users will either carry materials with them in a mobile lab of sorts (i.e. take the lab to field to some extent) or the instrument must carry its own supply of consumables. For fully automated systems there are two approaches for meeting this need. The first is to use liquid stocks that are held in containers and accessed by a series of valves. This method is used in commercially available, deployable instruments for nutrient analyses (e.g., SubChem Systems) and in the ESP. Alternatively, reagents can be dehydrated or encapsulated in expendable cartridges such as Cepheid's GeneExpert, Enigma Diagnostics' instruments and the AMG. Either way, the architecture of the instrument must accommodate reagents and other consumables (like filter media or probe arrays) that will be expended over time. Waste fluids will also be generated, and the instrument should have the capacity to store wastes onboard for proper 
disposal after retrieval. Consequently, it is desirable to keep reactions volumes as low as possible.

Beyond simply storing reagents and waste materials, reagents must also be stable for extended periods of time at ambient temperature and under prevailing atmospheric conditions under which they are deployed. Generally, molecular biological reagents are stored at a variety of temperatures (frozen, chilled, room temperature) and used in a normal air atmosphere. That luxury is difficult to accommodate in a field setting on an autonomous platform. Use of dehydrated reagent cocktails overcomes this limitation for some applications, though the reagents would have to remain very dry to be stable. In any case, stored reagents must tolerate swings in temperature and function under conditions that are novel to say the least. Various gases, including water vapor, may accumulate if the instrument is housed in a pressure vessel for subsurface operations. Over time, atmospheric conditions within the housing can change such that it no longer reflects normal air or dry nitrogen that might have been used to purge the vessel prior to deployment.

\section{Autonomous "ecogenomic sensors" for ocean research}

I am aware of only two examples where the steps of sample collection and molecular analytical analyses are integrated into an ocean-deployable instrument: the AMG and ESP (Paul et al., 2007). One could classify these instruments as "ecogenomic sensors" given my definition of that phrase, though they are nowhere near as highly evolved as that stylized in Fig. 1. Despite their limitations, the AMG and ESP at least provide a glimpse into the developments aimed at realizing systems for fielding molecular analytical techniques in a remote ocean setting. They also illustrate the maturity gap between these instruments versus the above mentioned commercial systems aimed at the biomedical, food safety and military markets.

The current prototype of the AMG collects water samples, filters cells and extracts nucleic acid using a series of expendable cartridges contained in a rotating carousel (Autonomous Microbial Genosensor). The AMG uses an isothermal RNA amplification technique referred to as nucleic acid sequencebased amplification, or NASBA. The same chemistry can be applied using a hand held device (Casper et al., 2007). The AMG is undergoing field trials at the time of this writing. Deployments are being conducted off the west cost of Florida with the primary target being detection of the red tide dinoflagellate, Karenia brevis (J. Paul, personal communication, 2009). This system and the chemistries it emulates are promising. One prototype of the AMG is operational and there are plans to develop additional units in the future. The technology readiness level (TRL) of the AMG is roughly 6-7 based on the US Department of Defense scale.
The functionality of the ESP parallels that of the AMG. It too uses a rotating carousel to store reactions chambers ("pucks") that house filter media and probe arrays. Pucks are loaded into various processing positions within the instrument using robotic mechanisms (Environmental Sample Processor; Roman et al., 2007; Scholin et al., 2009). Currently the ESP utilizes DNA probe and protein arrays to detect target molecules indicative of species and the substances they produce. The DNA arrays employ a sandwich hybridization method wherein rRNA is captured directly from a crude homogenate (Scholin et al., 2009). The protein arrays use a competitive ELISA methodology (Doucette et al., 2009). Application of the protein arrays follows the same basic steps and data telemetry as that for DNA arrays. An example of detecting changes in bacterioplankton rRNA sequences autonomously using the ESP and low density probe arrays is shown in Fig. 2. The ESP can also archive samples for laboratory analyses after the instrument is recovered, including fluorescent in situ hybridization (FISH), various nucleic acid analyses (cloning, sequencing) and algal toxin measurement (e.g., Greenfield et al., 2006, 2008; Doucette et al., 2009). The TRL of the core ESP is roughly 7-8. At the time of this writing (2009), five copies of the instrument are in operation and the system is commercially available through Spyglass Biosecurity in partnership with McLane Research Laboratories.

The ESP is meant to be modular system. It can be modified by adding external sampling modules to enable specialized sampling functions, and can accommodate a microfluidc module (or microfluidic block, MFB) for supporting small scale reactions downstream of sample collection and homogenization. Current sampling modules are designed such that the ESP can function in the deep-sea. Successful, short duration tests to $1000 \mathrm{~m}$ have been completed recently. The MFB is designed to accommodate separate molecular detection devices that require metering and moving fluids with $\mu \mathrm{l}$ accuracy and precision. Current work emphasizes a reusable solid phase extraction column for purifying nucleic acids and a 2-channel real-time PCR module. Refinement and testing of the deep-water sampling and MFB/PCR modules is ongoing. The deep-water sampling module has a TRL of 6 (a new $4 \mathrm{~km}$-rated version is in testing phase now). The MFB/PCR module has a TRL of 7-8 and is available commercially as a standalone unit for bench top testing or can be bundled with the core ESP for in situ operations.

\section{Recommendations}

Development of "ecogenomic sensors" can be enhanced through a variety of activities. Chief among them is creating dedicated teams composed of scientists, engineers and field operations specialists. The magnitude of the task at hand and the systems integration required demands a diverse work force that can focus on a development effort for an extended period of time. Second, there is a clear need to investigate 


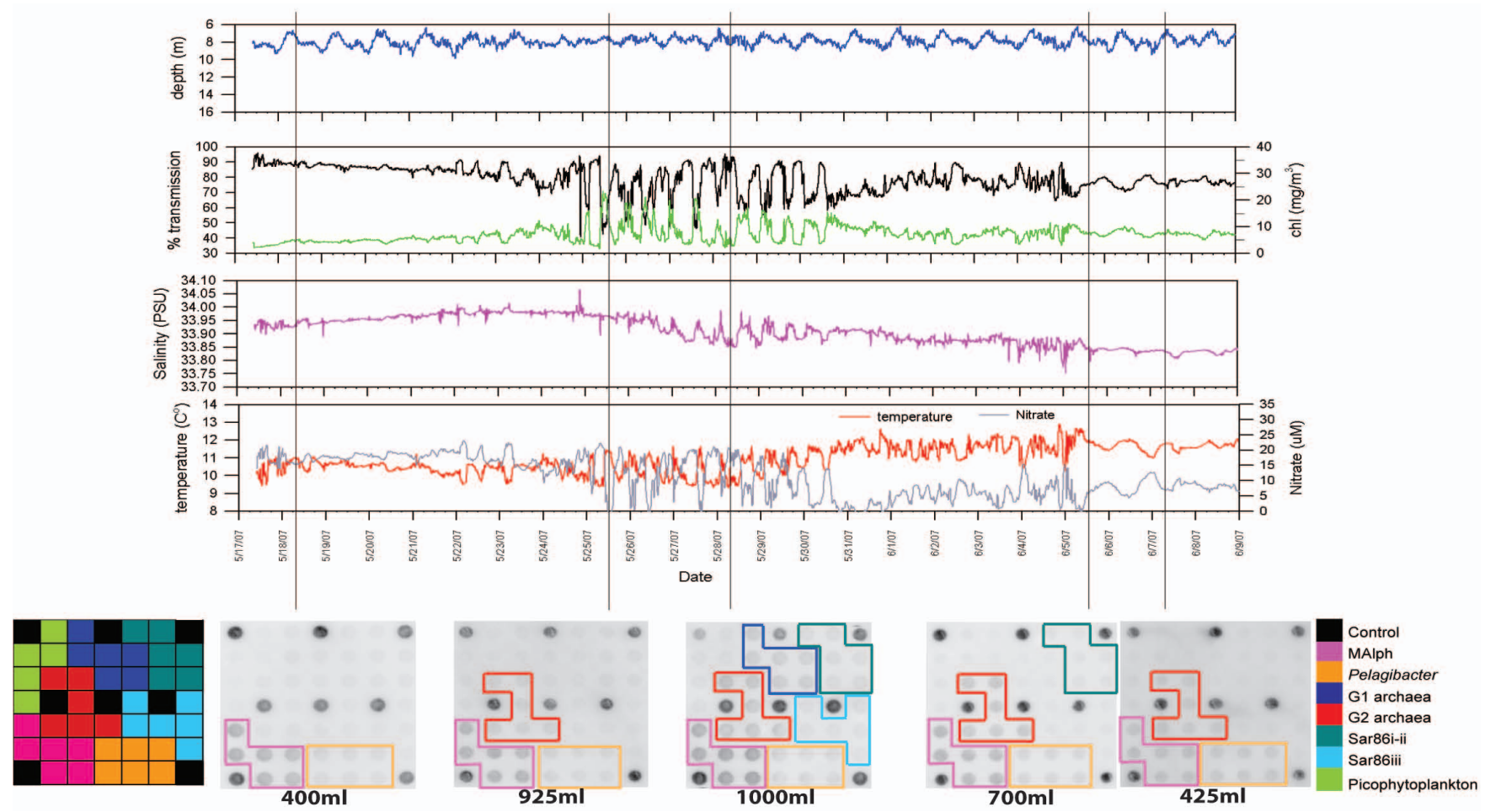

Fig. 2. Remote detection of marine bacterioplankton using ESP DNA probe arrays. The ESP was moored in Monterey Bay, California, 17 May-11 June, 2007. Top four graphs show physical and chemical data collected by contextual sensors on the ESP mooring during the deployment. The bottom images shows DNA probe arrays targeting rRNA indicative of various groups of marine bacterioplankton (after Preston et al., 2009); colored boxes at bottom left and right illustrate the array may and key, respectively. Sample volume is shown underneath the array. The images have been inverted such that positive reactions appear as dark spots on a white background. Marine alpha proteobacteria (MAlph) and Pelagibacter were present throughout the deployment. G2 euyarchaea appeared on 25 May and remained present though the end of the deployment. Between 25-30 May, relatively low nitrate, warm waters were followed by a pulse of upwelling characterized by high nitrate and low temperature. In the upwelled water, the ESP detected 6 of the 7 groups targeted including marine G1 crenarchaea (28 June). Groups highlighted on the arrays indicate positive signals that exceeded background +3 standard deviations. Actual size of the arrays shown are $\sim 15 \mathrm{~mm} \times 15 \mathrm{~mm}$; (Preston et al., unpublished data). During the same deployment the ESP also developed probe arrays for invertebrates and harmful algae. An example of the latter is shown here: http://www.mbari.org/ESP/field_data/2007/HAB_2007.htm. In the supplementary material animation showing basic operation of the ESP in surface waters (see http://www.ocean-sci.net/6/51/2010/ os-6-51-2010-supplement.zip).

"upstream" techniques for collecting and handling samples. The cutting edge molecular analytical detection technologies are clearly not well aligned with raw water samples and large volumes typical of environmental research. Third, instrument developments should bear in mind the need to detect multiple targets contained within single samples. There is a desire for visualizing many molecular signatures simultaneously and quantifying their interplay with changing environmental conditions. Some kind of array capability is needed and there are a variety of ways for meeting that requirement. Fourth, there is an expectation that ecogenomic sensors will function in large part as their laboratory counter parts do. That may work well for semi-automated systems that rely on people to handle materials and supplies, and where operations are conducted out of mobile labs largely free of the rigors imposed when instruments are deployed in the ocean.
But the same chemistries may not do well for in situ systems where conditions are less predictable (variable temperature, shock and vibration, etc.). So research teams should remain open minded as to detection chemistries that offer alternatives to the "norm", and not be wholly restricted by how something must be done given current dogma based on laboratory experience and industry standards. Fifth, system designs should consider options for sample archival. Preserved material can be used to help verify sensor functionality and serve the needs of basic research at that same time. Sixth, the advent of ecogenomic sensors places increased demands on data management and visualization tools. How one incorporates and displays molecular genetic data in 4$\mathrm{D}$ is not well defined. Likewise, metadata associated with the analyses (batch of reagents, quality control information) will need to be tracked to ensure that molecular signatures 
seen at one time can be compared directly to the same observed at another time/location by another sensor or team. Seventh, there is need for event response capability. Molecular analyses are inherently slow compared to most chemical and physical measurements. Even "rapid" tests by current standards can take 30 min or more when time for sample collection is included, and in the process each analytical cycle expends limited supplies of consumables (and power). Having the ability to trigger sampling events based on high frequency, low power assessments of the chemical and physical surroundings could greatly extend sensor deployment duration and reduce routine operations and maintenance costs. Lastly, results of whole-cell and cell-free assays applied to the same sample do not always yield the same impression as to what organisms are present (e.g., Scholin et al., 1999, 2000). Target cells may be free living, be embedded in detrital material, actively growing, dead or dying. Even if the target cell is eaten by another species its "biosignature" may persist for some period of time in food vacuoles or guts and even emerge in feces. The transfer of algal toxins in the food web is a vivid illustration of that point. Therefore, calibration of cell-free detection techniques relative to methods that rely on direct visualization of intact organisms poses many challenges with respect to the interpretation and operational application of molecular analytical data.

\section{Summary and conclusions}

Development of ecogenomic sensors remains a ripe area for future investigation from science, policy and systems engineering standpoints. Clearly, there are many options for realizing integrated molecular analytical sensing systems. But definition of key target molecules, detection methods and signal transduction modalities largely remain to be determined. The survey of approaches and companies provided here touch only a small subset of what is available from academic and commercial interests. Moreover, there remains a huge challenge of merging this new class of instrument with different deployment platforms, and supplying necessary power and data telemetry infrastructure for their operation. Ecogenomic sensors could find themselves on buoys, cabled observatories, profiling systems, near surface or great depth, easily accessible or in very remote locations, on piers or adjacent to waterways, or even in a mobile lab. Operations and maintenance requirements, and system costs, will vary accordingly and widely. Proxy measurements and modeling techniques could help interpolate between a discrete and limited set of sensors collecting data at a relatively low rate. Instruments designed for in situ use will benefit from an ability to apply multiple molecular analytical techniques to a single sample. One challenge in that regard is determining whether sample collection and handling requirements for a varied set of analyses are compatible. High biomass samples may be desired, even required, for direct detection assays like sandwich hybridization used in the ESP, but such concentrated samples might inhibit chemistries that employ amplification reactions such as PCR. Extraction techniques that might be "optimal" for DNA or rRNA may destroy mRNA. No doubt, much work remains to define the assays that will be deployed in situ and their concomitant, upstream sample collection and processing requirements. While some common themes will almost certainly emerge, it is highly doubtful that a single "ecogenomic sensor" will meet all of our perceived needs. In that light, systems engineering should be driven by clear set of science and/or monitoring objectives rather than what is possible to achieve technologically.

Edited by: T. Dickey

\section{References}

Agilent: information regarding production of custom microarrays, online available at: http://www.chem.agilent.com/en-US/ products/instruments/dnamicroarrays/pages/default.aspx, last access: November 2009.

Ahn, S., Kulis, D. M., Erdner, D. L., Anderson, D. M., and Walt, D. R: Fiber-Optic Microarray for Simultaneous Detection of Multiple Harmful Algal Bloom Species, Appl. Env. Microbiol., 72, 5742-5749, 2006

Anonymous: Charting the Course for Ocean Science in the United States for the next decade. An Ocean Research Priorities Plan and Implementation Strategy, NSTC Joint Subcommittee on Ocean Science and Technology, online available at: http: //www.sfos.uaf.edu/stratplan/ORPP-four_pager_032007.pdf, last access: November 2009, and http://ocean.ceq.gov/about/sup_ jsost_prioritiesplan.html, last access: June 2008, 26 January 2007.

Autonomous Microbial Genosensor: information regarding autonomous sensor system for deploying NASBA detection chemistry for environmental monitoring online available at: http: //www.marine.usf.edu/microbiology/genosensor.shtml, last access: November 2009.

Bavykin, S. G., Akowski, J. P., Zakhariev, V. M., Barsky, V. E., Perov, A. N., and Mirzabekov, A. D.: Portable system for microbial sample preparation and oligonucleotide microarray analysis, Appl. Env. Microbiol., 67, 922-928, 2001.

Belgrader, P., Elkin, C. J., Brown, S. B., Nasarabadi, S. N., Langlois, R. G., Milanovich, F. P., Colston Jr., B. W., and Marshall, G. D.: A reusable flow-through polymerase chain reaction instrument for the continuous monitoring of infectious biological agents, Anal. Chem., 75, 3446-3450, 2003.

Bowler, C., Karl, D. M., and Colwell, R. R.: Microbial oceanography in a sea of opportunity, Nature, 459, 180-184, 2009.

Bruckner-Lea, C. J., Stottlemyre, M. S., Holman, D. A., Grate, J. W., Brockman, F. J., and Chandler, D. P.: Rotating rod renewable microcolumns for automated, solid-phase DNA hybridization studies, Anal. Chem., 72, 4135-4141, 2000.

Casper, E. T., Patterson, S. S., Bhanushali, P., Farmer, A., Smith, M., Fries, D. P., and Paul, J. H.: A handheld NASBA analyzer for the field detection and quantification of Karenia brevis, Harmful Algae, 6, 112-118, 2007.

Cepheid: information regarding equipment and reagents for realtime PCR, including the GeneXpert system, online available at: http://www.cepheid.com/, last access: November 2009. 
Chandler, D. P., Brown, J., Bruckner-Lea, C. J., Olson, L., Posakony, G. J., Stults, J. R., Valentine, N. B., and Bond, L. J.: Continuous spore disruption using radially focused, highfrequency ultrasound, Anal Chem., 73, 3784-3789, 2001.

DeLong, E. F.: The microbial ocean from genomes to biomes, Nature, 459, 200-206, 2009.

DeLong, E. F., Preston, C. M., Mincer, T, Rich, V.: Community genomics among stratified microbial assemblages in the ocean's interior, Science, 311, 496-503, 2006.

Devereux, R., Rublee, P., Paul, J. H., Field, K. G., and Santo Domingo, J. W.: Development and applications of microbial ecogenomic indicators for monitoring water quality: Report of a workshop assessing the state of the science, research needs and future directions, Environ. Monit. Assess., 116, 459-479, 2006.

Dhadwal, H., Mukherjee, S. B., Kemp, P., Aller, J., Liu, Y., and Radway, J.: A dual detector capillary waveguide sensor for detection and quantification of hybridized target species, Anal. Chim. Acta, 598, 147-154, 2007.

Doney, S., Abbott, M. R., Cullen, J. J., Karl, D. M., and Rothstein, L.: From genes to ecosystems: the ocean's new frontier, Front. Ecol. Environ., 2, 457-466, 2004.

Doucette, G. J., Mikulski, C. M., Jones K. L., King, K. L., Greenfield D. I., Marin III, R., Jensen, S., Roman, B., Elliott, C. T., and Scholin, C. A.: Remote, subsurface detection of the algal toxin domoic acid onboard the Environmental Sample Processor: assay development and initial field trials, Harmful Algae, 8 , 880-888, 2009.

Duy, J. and Connell, L.: Fast and accurate detection of Alexandrium species using peptide nucleic acid probes and surface plasmon resonance, Fourth Symposium on Harmful Algae in the US, Woods Hole, MA, USA, Abstracts p. 103, 2007.

Ellison, C. K. and Burton, R. S.: Application of bead array technology to community dynamics of marine phytoplankton, Mar. Ecol. Prog. Ser., 288, 75-85, 2005.

Enigma Diagnostics: information regarding field portable and lab based real-time PCR systems, online available at: http://www. enigmadiagnostics.com/, last access: November 2009.

Environmental Sample Processor: information regarding autonomous sensor system for deploying molecular probe assays in the ocean, online available at: http://www.mbari.org/ESP/, last access: November 2009.

Frias-Lopez, J., Shi, Y., Tyson, G. W., Coleman, M. L., Schuster, S. C., Chisholm, S. W., and DeLong, E. F.: Microbial community gene expression in ocean surface waters, P. Natl. Acad. Sci. USA, 105, 3805-3810, 2008.

Fluidigm: information regarding equipment at consumables for real-time PCR arrays, online available at: http://www.fluidigm. com/, last access: November 2009

Fukuba, T., Yamamoto, T., Naganuma, T., and Fuji, T.: Microfabricated flow-through device for DNA amplification - towards in situ gene analysis, Chem. Eng. J., 101, 151-156, 2004.

Giovannoni, S. J., Hayakawa, D. H., Tripp, H. J., Stingl, U., Givan, S. A., Cho, J.-C., Oh, H.-M., Kitner, J. B., Vergin, K. L., and Rappe, M. S.: The small genome of an abundant coastal ocean methylotroph, Environ. Microb., 10, 1771-1782, 2008.

Goodwin, K. D. and Litaker, R. W.: Emerging technologies for monitoring recreational waters for bacteria and viruses, in: Oceans and Human Health Risks and Remedies from the Seas, edited by: Walsh, P. J., Smith, S. L., Fleming, L. E., Solo-
Gabriele, H. M., and Gerwick, W. H., Academic Press, London, 381-404, 2008.

Greenfield, D. I., Marin III, R., Jensen, S., Massion, E., Roman, B., Feldman, J., and Scholin, C.: Application of the Environmental Sample Processor (ESP) methodology for quantifying Pseudonitzschia australis using ribosomal RNA-targeted probes in sandwich and fluorescent in situ hybridization, Limnol. Oceanogr., Methods, 4, 426-435, 2006.

Greenfield, D., Marin III, R., Doucette, G. J., Mikulski, C., Jensen, S., Roman, B., Alvarado, N., and Scholin, C. A.: Field applications of the second-generation Environmental Sample Processor (ESP) for remote detection of harmful algae: 2006-2007, Limnol. Oceanogr., Methods, 6, 667-679, 2008.

Gyros: information regarding equipment and consumables for micro-immunoassays on a compact disc format, online available at: http://www.gyros.com/, last access: November 2009.

Haywood, A. J., Scholin, C. A. , Marin III, R., Steidinger, K. A., Heil, C. A., and Ray, J.: Molecular detection of the brevetoxinproducing dinoflagellate Karenia brevis (Dinophyceae) and closely related species using ribosomal RNA probes and a semiautomated sandwich hybridization assay, J. Phycol., 43, 12711286, 2007.

Idaho Technology: information regarding equipment at consumables for automated pathogen identification and DNA analysis, online available at: http://www.idahotech.com/, last access: November 2009

Illumina: information regarding equipment and consumables for array-based DNA, RNA and protein analysis, online available at: http://www.illumina.com/, last access: November 2009.

Jones, W. J., Preston, C., Marin III, R., Scholin, C., and Vrijenhoek, R.: A Robotic Molecular Method for in situ Detection of Marine Invertebrate Larvae, Molec. Ecol. Res., 8, 540-550, 2007.

LaGier, M. J., Fell, J. W., and Goodwin, K. D.: Electrochemical detection of harmful algae and other microbial contaminants in coastal waters using hand-held biosensors, Mar. Pollut. Bull., 54, 757-770, 2007.

Luminex Corporation: information regarding equipment and consumables for flow cytometric-based, microbead array detection systems, online available at: http://www.luminexcorp.com/, last access: November 2009.

Lyons, M. M., Lau, Y., Carden, W. E., Ward, J. E., Roberts, S. B., Somolowitz, R., Vallino, J., and Allam, B.: Characteristics of marine aggregates in shallow water ecosystems: Implications for disease ecology, EcoHealth, 4, 406-420, 2007.

Metfies, K., Töbe, K., Scholin, C., and Medlin, L. K.: Laboratory and field applications of ribosomal RNA probes to aid the detection and monitoring of harmful algae, in: Ecology of Harmful Algae, edited by: Granéli, E. and Turner, J. T., Springer Verlag, Berlin, 311-325, 2006.

microfluidic ChipShop: information regarding equipment at consumables for "lab-on-chip", microfluidic systems, online available at: http://www.microfluidic-chipshop.com/, last access: November 2009.

OceanSensors08: workshop for marine scientists, sensor developers and technologists with an interest in determining and shaping the future of ocean sensing; white papers available under "Documents" section, online available at: http://www.io-warnemuende.de/conferences/oceans08/ content.php?mode=whitepapers, last access: November 2009. 
Ottesen, E. A., Hong, J. W., Quake, S. R., and Leadbetter, J. R.: Microfluidic digital PCR enables multigene analysis of individual environmental bacteria, Science, 314, 1464-1467, 2006.

Pall Corporation: Introduction to Tangential Flow Filtration for Laboratory and Process Development Applications, online available at: http://www.pall.com/laboratory_34212.asp, last access: November 2009.

Palmer, C. J., Bonilla, J. A., Bonilla, T. D., Goodwin, K. D., Elmir, S. M., Abdelzaher, A. M., and Solo-Gabriele, H. M.: Future of Microbial Ocean Water Quality Monitoring, in: Oceans and Human Health Risks and Remedies from the Seas, edited by: Walsh, P. J., Smith, S. L., Fleming, L. E., Solo-Gabriele, H. M., and Gerwick, W. H., Academic Press, London, 405-421, 2008.

Paul, J., Scholin, C., van den Engh, G., and Perry, M. J.: In situ Instrumentation, Oceanography, 20, 58-66, 2007.

Preston, C., Marin III, R., Jenson, S., Feldman, J., Massion, E., DeLong, E., Suzuki, M., Wheeler, K., Cline, D., Alvarado, N., and Scholin, C.: Near real-time, autonomous detection of marine bacterioplankton on a coastal mooring in Monterey Bay, California, using rRNA-targeted DNA probes, Environ. Microbiol., 11, 1168-1180, 2009.

Ramsdell, J. S., Anderson, D. M., and Glibert, P. M.: Harmful Algal Research and Response: A National Environmental Science Strategy 2005-2015, Ecological Society of America, Washington, DC, $96 \mathrm{pp}$, online available at: http://www.whoi.edu/ redtide/page.do?pid=15075 (last access: November 2009), 2005.

Rich, V. I., Konstantinidis, K., and DeLong, E. F.: Design and testing of 'genome-proxy' microarrays to profile marine microbial communities, Environ. Microbiol., 10, 506-521, 2007.

Roman, B., Scholin, C., Jensen, S., Massion, E., Marin III, R., Preston, C., Greenfield, D., Jones, W., and Wheeler, K.: Controlling a Robotic Marine Water Sampler with the Ruby Scripting Language, Journal of American Laboratory Automation, 12, 56-61, 2007

Sandifer, P., Sotka, C., Garrison, D., and Fay, V.: Interagency Oceans and Human Health Research Implementation. Plan: A Prescription for the Future, Interagency Working Group on Harmful Algal Blooms, Hypoxia, and Human Health of the Joint Subcommittee on Ocean Science and Technology, Washington, DC, online available at: http://www.whoi.edu/cms/files/ COHH-impleplan_31823.pdf, (last access: November 2009), 2007.

Scholin, C., Jensen, S., Roman, B., Massion, E., Marin III, R., Preston, C., Greenfield, D., Jones, W., and Wheeler, K.: The Environmental Sample Processor (ESP) - An Autonomous Robotic Device for Detecting Microorganisms Remotely using Molecular Probe Technology, Proceedings, OCEANS 2006 MTS/IEEE Conference, Boston, MA, Marine Technology Society, Columbia, MD, 2006.

Scholin, C. A., Doucette, G. J. and Cembella, A. D.: Prospects for developing automated systems for in situ detection of harmful algae and their toxins, in: Real-Time Coastal Observing Systems for Ecosystem Dynamics and Harmful Algal Blooms, edited by: Babin, M., Roesler, C. S., and Cullen, J. J., UNESCO Publishing, Paris, France, 413-462, 2008.
Scholin, C., Doucette, G., Jensen, S., Roman, B., Pargett, D., Marin III, R., Preston, C., Jones, W., Feldman, J., Everlove, C., Harris, A., Alvarado, N., Massion, E., Birch, J., Greenfield, D., Vrijenhoek, R., Mikulski, C., and Jones, K.: Remote detection of marine microbes, small invertebrates, harmful algae and biotoxins using the Environmental Sample Processor (ESP), Oceanography, 22, 158-167, 2009.

Scholin, C. A., Gulland, F., Doucette, G., et al.: Mortality of sea lions along the central California coast linked to a toxic diatom bloom, Nature, 403, 80-84, 2000.

Scholin, C. A., Vrieling, E., Peperzak, L., Rhodes, L., and Rublee, P.: Detection of HAB species using lectin, antibody and DNA probes, in: Manual on Harmful Marine Microalgae, edited by: Hallegraeff, G. M., Anderson, D. M., and Cembella, A. D., Intergovernmental Oceanographic Commission, UNESCO, Paris, Vol. 11. Second Edition, 131-164, 2003.

Scholin, C., Marin, R., Miller, P., Doucette, G., Powell, C., Howard., J., Haydock, P., and Ray, J.: Application of DNA probes and a receptor binding assay for detection of Pseudonitzschia (Bacillariophyceae) species and domoic acid activity in cultured and natural samples, J. Phycol., 35, 1356-1367, 1999.

STMicroelectronics: information regarding lab-on-chip system that combines PCR and low density probe array, online available at: http://www.st.com/stonline/products/technologies/labonchip/ technolo.htm, last access: November 2009.

Straub, T. M., Dockendorff, B. P., Quionez-Díaz, M. D., et al.: Automated methods for multiplexed pathogen detection, J. Microbiol. Methods, 62, 303-316, 2005.

SubChem Systems: information regarding autonomous chemical sensors for environmental research, online available at: http: //www.subchem.com/, last access: November 2009.

Varshney, M., Li, Y., Srinivasan, B., and Tung, S.: A labelfree, microfluidics and interdigitated array microelectrode-based impedance biosensor in combination with nanoparticles immunoseparation for detection of Escherichia coli O157:H7 in food samples, Sensors and Actuators B, 128, 99-107, 2007.

Venter, J. C., Remington, K., Heidelberg, J. F., et al.: Environmental genome shotgun sequencing of the Sargasso Sea, Science, 304 66-74, 2004.

Technology Readiness Level: A numbering system for identifying maturity of evolving systems; more information is, online available at: http://en.wikipedia.org/wiki/Technology Readiness_Level, last access: November 2009

Zehr, J. P., Hewson, I., and Moisander, P.: Molecular biology techniques and applications for ocean sensing, Ocean Sci., 5, 101113,2009 .

http://www.ocean-sci.net/5/101/2009/. 